\title{
Cytogenomic Abnormalities and Underlying Mechanisms for Intellectual and Developmental Disabilities
}

\section{Peining $\mathrm{Li}^{*}$}

Department of Genetics, Yale School of Medicine, New Haven, CT, USA

The first decade post Human Genome Project has been marked with waves of emerging high-throughput genomic technologies and their immediate impact on genetic research and service. The first wave of genome-wide oligonucleotide array comparative genomic hybridization (aCGH) and single nucleotide polymorphism (SNP) chips have resulted in the discovery of copy number variants (CNVs) in the human genome and the evolution of clinical cytogenetics into cytogenomics. CNVs are defined as the gain or loss of genomic materials larger than $1 \mathrm{~kb}$ in size and they present in approximately $12 \%$ of the genome in normal human populations [1]. A CNV detected from a normal individual is likely a polymorphic variant without clinical significance and usually termed as a benign CNV (bCNV). A $\mathrm{CNV}$ with known disease association is referred as pathogenic CNV (pCNV), and a rare or private $\mathrm{CNV}$ with uncertain clinical relevance is named a variant of unknown significance (VUS) [2]. The clinical application of aCGH and SNP chips on pediatric patients with intellectual and developmental disabilities (ID/DD) provides a better diagnosis of chromosomal and genomic imbalances with delineated genomic coordinates and defined gene content. Accumulated cases with similar or overlapped genomic imbalances facilitate fine-mapping of critical regions harboring dosage-sensitive genes for accurate 'genotype-phenotype' correlation. Functional analyses using in vitro cellular phenotyping and in vivo animal modeling have been developed for clinically detected pCNVs. For many newly detected pCNVs, there is urgent demand for rapid transition from diagnostic discovery to study of disease-causing mechanisms and exploration of therapeutic approaches.

\section{Cytogenomic Abnormalities in Intellectual and Developmental Disabilities}

ID/DD occurs in 1 3\% of the general population. Based on many validation reports and experts' consensus, current practice guidelines from the American College of Medical Genetics (ACMG) recommend that aCGH and SNP chip analysis should be the first-tier genetic testing, replacing classic cytogenetic analysis, for pediatric patients with ID/DD, multiple congenital anomalies (MCA), autism spectrum disorders (ASD), speech and language delay, schizophrenia and epilepsy. Although classic cytogenetic analysis has gradually become a supplemental or confirmatory procedure, karyotyping is still the gold standard to detect numerical chromosomal abnormalities (e.g., trisomy 21 for Down syndrome and monosomy X for Turner syndrome) and structural rearrangements (e.g. Robertsonian translocation). Fluorescent in situ hybridization (FISH) is also the 'cellbased' method of choice to determine mosaic patterns. Approximately $45 \%$ of genomic imbalances are larger than 3-5 Mb and could be confirmed by high resolution G-banding; most recurrent genomic disorders, subtelomeric rearrangements and mosaic patterns can be readily confirmed by clinically-validated commercial FISH probes. The integrated cytogenomic analysis has significantly improved the diagnostic yield from $3.7-6.8 \%$ by molecular cytogenetic analysis to 12 -
$20 \%$ by oligonucleotide aCGH or SNP gene chip [3]. From 2006-2011, there were 1,354 consecutive pediatric patients analyzed by $44 \mathrm{~K}$ and $180 \mathrm{~K}$ oligonucleotide aCGH (Agilent) in the Yale Cytogenetics and Genomics Laboratory, and pathogenic abnormalities were detected in 176 patients (13\% diagnostic yield). These abnormalities were classified into chromosomal and cryptic structural abnormalities in 95 cases (54\%), recurrent genomic disorders in 66 patients $(37.5 \%)$, and common aneuploidies in 15 patients $(8.5 \%)$.

Widely accepted clinical application of aCGH and SNP chips has rapidly accumulated a large amount of cytogenomic abnormalities from diagnostic cytogenetic and molecular laboratories. The spectrum of these abnormalities ranges from large interstitial/subtelomeric imbalances and submicroscopic recurrent genomic disorders to cryptic and intragenic copy number aberrations. The collection of bCNVs, pCNVs and VUSs into web-delivered databases has provided an essential tool in interpreting results for diagnostic laboratories and also in educating clinical geneticists and genetic counselors. The Database of Genomic Variants (DGV), International Standards for Cytogenomic Arrays (ISCA), DatabasE of Chromosomal Imbalance and Phenotype in Humans using Ensembl Resources (DECIPHER) and other related web resources have been loaded onto the Human Genome Browser as searchable tracks. The brief clinical description from DECIPHER and the evidence-based rating of CNV into pathogenic, likely pathogenic, uncertain significance, likely benign and benign from ISCA are all helpful in reporting genomic findings. Recognized cytogenomic syndromes have entries in the Online Mendelian Inheritance in Man (OMIM). Novel cytogenomic abnormalities are usually presented as case reports and can be searched from the PubMed. Reports of a series of similar findings and in-depth reviews will provide multiple lines of evidence for 'genotype-phenotype' correlation.

Genomicdelineation of chromosomallyobserved complexstructural rearrangements and heterogeneous interstitial and subtelomeric imbalances allows fine-mapping of critical regions or intervals to identify potential candidate dosage-sensitive genes [4,5]. Cytogenomic analysis also resolves the genomic structures, mutagenesis mechanisms and mitotic or meiotic behaviors from puzzling chromosomal structural abnormalities like ring chromosomes or supernumerary marker chromosomes. The findings of unique deletion and duplication

*Corresponding author: Peining Li, Department of Genetics, Yale School of Medicine, New Haven, CT, USA, E-mail: peining.li@yale.edu

Received July 16, 2013; Accepted July 17, 2013; Published July 22, 2013

Citation: Li P (2013) Cytogenomic Abnormalities and Underlying Mechanisms for Intellectual and Developmental Disabilities. J Mol Genet Med 7: 73 doi:10.4172/1747-0862.1000073

Copyright: () $2013 \mathrm{Li} \mathrm{P}$. This is an open-access article distributed under the terms of the Creative Commons Attribution License, which permits unrestricted use, distribution, and reproduction in any medium, provided the original author and source are credited 
patterns and distinct mitotic behavior in ring chromosome 21 supported further sub-typing of ring chromosomes for better clinical association [6]. Recurrent microdeletions and microduplications, also termed genomic disorders, are mediated by non-allelic homologous recombination within regional low copy repeats (LCRs). Genomic disorders such as DiGeorge syndrome (OMIM\#188400) caused by a deletion at 22q11.2, Williams-Beuren syndrome (OMIM\#194050) by a deletion at 7q11.23, Prader-Willi syndrome (OMIM\#176270) and Angelman syndrome (OMIM\#105830) by a deletion at $15 q 11.2$ have been recognized clinically and routinely diagnosed by FISH testing. The aCGH and SNP chip analyses detect precisely these previouslydefined genomic disorders and also many new genomic disorders. A recent study on a large cohort of 15,767 pediatric patients with ID/DD indicated that about $14.2 \%$ of them are caused by pCNVs over $400 \mathrm{~kb}$, and approximately $60 \%$ of these pCNVs are within 45 known genomic disorder regions [7]. The most commonly seen 14 genomic disorders are mapped at 22q11.2, 16p11.2, 1q21.1, 15q13.2-q13.3, 7q11.23, 15q11.2-q13, 17q21.31, 16p13.11, 17p11.2, 17q12, 1q21, 8p23.1, 5q35 and 3q29; collectively they represent a $4.5 \%(1 / 22)$ diagnostic yield in pediatric patients and an estimated $0.18 \%(1 / 550)$ prevalence in the general population [3]. The diagnosis of these genomic disorders has become an integral part of pediatric genetic evaluation.

\section{Dissecting Dosage-Sensitive Genes and Disease Causing Mechanisms}

For many newly detected pCNVs, little is known about the dosagesensitive genes and their cellular and developmental functions. The limited availability and accessibility of live brain and neuron tissues is the major obstacle in the study of disease-causing mechanisms in human mental development. Recent progress in stem cell technologies has made possible the modeling of human mental diseases using patient derived stem cells. In 2010, Marchetto et al. developed a culture system using induced pluripotent stem cells (iPSCs) from Rett syndrome patients' fibroblasts [8]. These Rett syndrome iPSCs were able to undergo $\mathrm{X}$-inactivation and generate functional neurons. Neurons derived from these iPSCs had fewer synapses, reduced spine density, smaller soma size, altered calcium signaling and electrophysiological defects when compared to controls. This cellular model provided critical evidence of an unexplored developmental window before disease onset and enable direct testing of drug effect in rescuing synaptic defects.

The microdeletion and microduplication at the same genomic locus offer an opportunity to study dosage-sensitive genes, especially for the opposite phenotypes of haploinsufficient and triple-sensitive genes. However, clinical evaluation could be complicated by overlapped phenotypes, variable expressivity, reduced penetrance and lack of longitudinal study of late-onset phenotypes for many genomic disorders. Recent studies observed opposite phenotypes in a few genomic disorders. For example, the microdeletion syndrome at 16p11.2 (OMIM\#611913) and the reciprocal microduplication syndrome (OMIM\#614671) were initially associated with ASD but a subsequent study revealed mirror body mass index phenotypes. Microdeletion at $16 \mathrm{p} 11.2$ is often associated with obesity, macrocephaly and ASD, while the reciprocal microduplication is associated with underweight, microcephaly and schizophrenia [9]. Mouse models of 16 p11.2 microdeletion and microduplication detected in vivo brain anomalies and behavior disorders [10]. Overexpression and transcript suppression of the 29 candidate genes from this $16 \mathrm{p} 11.2$ region in zebra fish identified the KCTD13 gene as a major driver of the mirrored neuroanatomical phenotypes [11]. Although the physiology and genetic makeup in the model animals are different from that of humans, the animal models allow direct evaluation of gene-dosage effects and association of neuroanatomical defects with phenotypes.

\section{From Benchside Dignostic Discovery to Bedside Treatment}

The diagnostic application of aCGH or SNP chips has contributed greatly to our understanding of genetic etiology in $12 \%-20 \%$ of pediatric patients with $\mathrm{DD} / \mathrm{ID} / \mathrm{MCA} / \mathrm{ASD}$. This experience revealed a systematic approach, including the analytical and clinical validation of novel high-throughput genomic technologies, the establishment of clinical evidence by large scale case-control studies and the development of practice standards and guidelines, for the integration of the nextgeneration whole-genome exome sequencing and the next-next generation genomic sequencing technologies into clinical screening and diagnosis. However, the increased technical complexity of exome or genomic sequencing and the intellectual challenge to interpret a large amount of sequencing data will require more collaborative effort and supportive resources. The ultimate goal for an integrated pediatric genetic diagnosis is to provide comprehensive and in-depth profiling of karyotype, pCNV and mutations and associated medical phenotypes and risks for patients. Furthermore, the implementations of knowledge-based genetic counseling, rational clinical action and follow up familial studies could be of direct benefit for a substantial proportion of patients. For example, the aggressive behavior from patients with a 15q13.3 deletion involving the CHRNA7 gene could benefit from treatment with the NChR allosteric modulator and acetylcholinesterase (AChE) inhibitor, galantamine [12]. As we gain more knowledge of these genomic abnormalities through functional analysis and therapeutic trials using in vitro cellular and in vivo animal models, disease-specific guidance management and treatment could be developed, culminating in fully personalized medicine.

\section{References}

1. Redon R, Ishikawa S, Fitch KR, Feuk L, Perry GH, et al. (2006) Global variation in copy number in the human genome. Nature 444: 444-454.

2. Lee C, lafrate AJ, Brothman AR (2007) Copy number variations and clinical cytogenetic diagnosis of constitutional disorders. Nat Genet 39: S48-54.

3. Wei Y, Xu F, Li P (2013) Technology-driven and evidence-based genomic analysis for integrated pediatric and prenatal genetic evaluation. J Genet Genomics 40: 1-14.

4. Li P, Zhang HZ, Huff S, Nimmakayalu M, Qumsiyeh M, et al. (2006) Karyotype-phenotype insights from 11q14.1-q23.2 interstitial deletions: FZD4 haploinsufficiency and exudative vitroretinopathy in a patient with a complex chromosome rearrangement. Am J Med Genet 140A: 2721-2729.

5. Rossi MR, DiMaio MS, Xiang B, Lu K, Kaymakcalan H, et al. (2009) Clinical and genomic characterization of distal duplications and deletions of chromosome 4q: Study of two cases and review of the literature. Am J Med Genet 149A: 2788-2794.

6. Zhang HZ, Xu F, Seashore M, Li P (2012) Unique genomic structure and distinct mitotic behavior of ring chromosome 21 in two unrelated cases. Cytogenet Genome Res 136: 180-187.

7. Cooper GM, Coe BP, Girirajan S, Rosenfeld JA, Vu TH, et al. (2011) A copy number variation morbidity map of developmental delay. Nat Genet 43: 838846.

8. Marchetto MC, Carromeu C, Acab A, Yu D, Yeo GW, et al. (2010) A mode 
Citation: Li P (2013) Cytogenomic Abnormalities and Underlying Mechanisms for Intellectual and Developmental Disabilities. J Mol Genet Med 7: 73. doi:10.4172/1747-0862.1000073

for neural development and treatment of Rett syndrome using human induced pluripotent stem cells. Cell 143: 527-539.

9. Jacquemont S, Reymond A, Zufferey F, Harewood L, Walters RG, et al. (2011) Mirror extreme BMI phenotypes associated with gene dosage at the chromosome 16p11.2 locus. Nature 478: 97-102.

10. Horev G, Ellegood J, Lerch JP, Son YE, Muthuswamy L, et al. (2011) Dosagedependent phenotypes in models of 16p11.2 lesions found in autism. Proc Natl Acad Sci USA 108: 17076-17081.
11. Golzio C, Willer J, Talkowski ME, Oh EC, Taniguchi Y, et al. (2012) KCTD13 is a major driver of mirrored neuroanatomical phenotypes of the 16p11.2 copy number variant. Nature 485: 363-367.

12. Cubells JF, Deoreo EH, Harvey PD, Garlow SJ, Garber K, et al. (2011) Pharmaco-genetically guided treatment of recurrent rage outbursts in an adult male with 15q13.3 deletion syndrome. Am J Med Genet A 155: 805-810. 